\title{
SOME POINTS IN THE TREATMENT OF ENLARGED PROSTATE.
}

\author{
By LEVESON-GOWER GUNN, M.D. UNIT. 1)rB., F.R.C.S.; \\ Assistant Surgeon to the Adelaide Hospital, Dublin.
}

[Read in the Section of Surgery, January 11, 1907.]

IT is hardly possible to read the current British and American medical journals without finding every week one or more papers on the treatment of enlarged prostate; most of these seem to have been written with this purpose-to demonstrate that the author's plan of operating on the gland is the best method, and that he and he only was the inventor of the operation.

It seems to be taken for granted that some form of operation is the only possible treatment. The non-operative treatment is seldom mentioned, ret I venture to think that there are more patients at the present time treated by non-operative measures for enlarged prostates, and with a large measure of success, than there are patients who are treated by operation.

To-night I wish to deal with the broad general lines, rather than with the details of treatment; to aroid any discussion as to which operative procedure is the most useful: to remain silent on operative technique, for these points are to the surgeons present already familiar, and to those who are not engaged in operative work their rerital can only be wearisome.

During the past two years I have had some opportunity of seeing enlargement of the prostate treated at three different centres of learning - at London, at Paris, and at Berlin-on diverse lines, by men who had specialised at 
genito-urinary work. I propose now to give you an outline of these three methods, and then, very briefly, to tell you the plan I try to carry out myself in the treatment of such cases.

At Professor Hartmann's clinic the diagnosis was made from the symptoms, the urine examined for pus and albumin, a rectal examination, sometimes followed by the passage of a soft catheter to find out the amount of residual urine. The treatment is carried out on general hygienic lines; the patient is handed a paper bearing the following instructions:- "Wine, beer, liqueurs, coffee, tea, all spicy foods, tomatoes, and asparagus must be aroided. Take a teaspoonful of bicarbonate of soda and dissolve this in a litre of filtered water: take a wineglass of this at earh meal. Take short, brisk walks: do not remain more than seven hours in bed: let the bed be a hard one. Make each morning a dry friction with a rough $r \cdot \operatorname{loth}$ on which has been sprinkled a few drops of spirit or eau de rologne over the entire surface of the body. Do not try to pass water oftener than is natural."

As long as the patient can pass water nothing further is done unless the urine contains pus and the patient shows signs of septic absorption. Under these circumstances a soft catheter is passed daily and the bladder washed out with 3000 oxycyanide of $\mathrm{Hg}$. Should the patient be unable to pass water, he is taught to clean and pass a gum elastic catheter, and told to mash out the bladder each time the instrument is passed.

The male patients attending this clinic averaged about 150 per diem. A fair proportion of these cases suffered from enlarged prostate, yet during four weeks there I saw no case advised to have his prostate removed.

In Berlin, at Frank's clinic, the number of patients was not so large, but the methods of examination were far 
more thorough. The symptoms were taken in the usual way, the urine submitted to a careful micro- and macroscopic examination; if bacteria were present, they were, as a rule, planted out. The rectal examination was follored by a cystoscopic examination of the interior of the bladder, the amount of residual urine being moasured at the same time.

The treatment in cases where the urine was aseptic consisted in passing occasionally, about once a month, a fullsized metal bougie and leaving this distending the prostatic urethra for five minutes. Purgatives were freely given, and great stress was laid on this, but no attempt was made to stop beer or alcohol.

If the obstructive symptoms were severe prostatectomy was advised in all cases with aseptic urine.

In cases with septic urines a partial operation, such as Bottini's, ras performed.

If the sepsis was rery severe the bladder was washed out by catheter for a few days, and then perineal drainage was established.

Of the treatment adopted in London I can speak with less certainty, as the time there at my disposal was very limited. The diagnosis was made by taking the symptoms and a rectal examination; microscopic examination of the urine did not seem to be the rule. The residual urine was tested by passing a soft catheter.

The treatment recommended in every case seemed to be prostatectomy.

In looking at these three methods of treatment I felt that a less severe treatment than that adopted at London might have sufficed in some of the cases, that the Berlin method of treating the more septic cases did not seem satisfactory, while some of the Paris cases would undoubtedly have been benefited by an operation. 
I try to apply the following principles in the treatment of enlarged prostate:-

Let the examination of the patient be as complete as possible. Examine the urine macroscopically and microscopically, the rectum, the urethra, and the interior of the bladder, by this finding out the extent the gland is enlarged up into the bladder, the appearance of the prostate, whether suggestive of malignancy or not, the presence of calculi, the condition of the bladder walls, if sacculated, the discharge of pus from either ureter, for on these findings the treatment of the case must largely depend

In mild cases, where the bladder wall is healthy, where the urine is aseptic and the patient able to pass water, though not to empty his bladder, I stop all alcohol, keep the bowels free, promote the healthy action of the skin, warn the patient against exposing himself to wet or cold, pass once a month, if necessary, a full-sized metal bougie. In such cases the indications for operation would be-the pain being severe, the presence of calculi, the possibility of the gland being malignant. In cases where a mild chronic cystitis is present $I$ adopt the same treatment with the addition of internal urinary antiseptics. The indications here for operation are sacculation of the bladder or signs of infection spreading to the kidneys.

In septic cases which require catheterisation I wash the bladder for a week before doing a prostatectomy; the contra-indications to operation are-extreme age, great weakness, or a double pyelitis. In acutely septic cases with much ferer I drain the bladder for a few days before doing a radical operation, and give stimulants freely during this time.

It is possible you may think from my remarks that $I$ am not in farour of prostatectomy. This is not the case. 
The operation is often admirable in its result, and in many cases is the only way in which a patient can be relleved from his distress, but at the same time I do think that many medical men hold mistaken ideas about this operation of prostatectomy. It is looked upon as an operation easy to perform, uniformly successful in its results, and having almost no mortality. Quite lately a patient informed me that his doctor told him that the operation consisted in making a small opening into the bladder and picking the gland off the floor, the operation only lasting a fer minutes. This gentleman had not seen, as I have, a urinary surgical specialist struggle for forty minutes before he succeeded in "picking the gland off the floor of the bladder." Again, after this operation a stricture may form, or if the compressor urethra is injured incontinence may occur, learing the patient in a very miserable condition. A supra-pubic or perineal fistula, as the case may be, sometimes persists for a long time, and causes much discomfort. Lastly, as to the risks of the operation, I would put the mortality at about $\delta$ to 10 per cent., in spite of lengthy statistics showing almost no mortality. When I think of the age at which this operation is usually performed, the amount of sepsis often present, and the extent to which the patient's kidneys are sometimes diseased, it seems impossible to me that prostatectomy should not have a considerable mortality, and this mortality must be borne in mind in recommending a patient to submit to the operation. On the other hand, I do not think that the risks of a prostatectomy can ever be so great as those incurred by the constant use of a catheter.

Mr. Walton Browne (Belfast) was pleased to hear Mr. Gunn advocating not being in a hurry in dealing with such 
cases. He believed a good deal could be done by washing out the bladder.

MR. C. A. K. BALL expressed the view that a large number of cases had been operated on in the past which, no doubt, would have lived, and perhaps in a more comfortable state, had palliative measures been tried instead of operation. He thought that palliative measures ought to be employed unless there was absolute obstruction to the flow of urine.

Mr. D. KENNEDY thought that before recommending either palliative or radical treatment the circumstances of the patient should be taken into consideration. A well-to-do patient could afford to avail himself of skilled surgical aid, and might well decide to undergo treatment of a palliative nature; but in the case of a man without means the proper employment of a catheter or other palliative measures might be attended with difficulty.

MR. BLAYNEY considered that there was a certain amount of danger attending the return to more conservative measures in the treatment of enlarged prostate. Many cases which had ended fatally after operation would probably have recovered had radical measures been carried out earlier. 